\title{
miR-183 inhibits microglia activation and expression of inflammatory factors in rats with cerebral ischemia reperfusion via NF-кB signaling pathway
}

\author{
BO XIANG ${ }^{1}$, PING ZHONG ${ }^{1}$, LEI FANG ${ }^{1}$, XIANXIAN WU ${ }^{1}$, YUQIANG SONG $^{2}$ and HAICHENG YUAN ${ }^{1}$ \\ ${ }^{1}$ The Second Department of Neurology, Qingdao Central Hospital, Qingdao, Shandong 266042; \\ ${ }^{2}$ Department of Neurology, The Affiliated Hospital of Qingdao University, Qingdao, Shandong 266003, P.R. China
}

Received October 19, 2018; Accepted June 13, 2019

DOI: $10.3892 /$ etm.2019.7827

\begin{abstract}
Ischemic stroke represents $87 \%$ of all strokes, and is the third leading cause of disability and mortality worldwide. The cause of ischemic stroke is the obstruction of blood flow through the artery that supplies oxygen-rich blood to the brain, with ischemia-reperfusion injury as its major cause. microRNAs (miRNA) are small non-coding RNAs, which serve important roles in the regulation of gene expression at the post-transcription level. The aim of the present study was to investigate the effect of miRNA-183 (miR-183) on microglia activation in rats with cerebral ischemia-reperfusion injury. To this end, a rat cerebral ischemia-reperfusion injury model was established. The results indicated that miR-183 expression was decreased by cerebral ischemia-reperfusion. In addition, treatment using miR-183 agomir significantly reduced the neurological function scores, percentage of cerebral infarction volume, and ionized calcium-binding adapter molecule-1 (IBA-1)-positive cells in the CA1 area of the hippocampus in rats subjected to cerebral ischemia-reperfusion injury, implicating a neuroprotective role for miR-183. MiR-183 agomir treatment also decreased the expression of pro-inflammatory-associated proteins interleukin (IL)-1 $\beta$, IL-6 and tumor necrosis factor (TNF)- $\alpha$. Finally, the expression of the nuclear factor (NF)- $\kappa \mathrm{B}$ p 65 and $\mathrm{I} \kappa \mathrm{B} \alpha$ was decreased and increased by miR-183 agomir treatment, respectively, indicating inhibition of the NF- $\mathrm{NB}$ signaling pathway. These observations suggest that miR-183 regulates the activation of microglia in rats with cerebral ischemia-reperfusion injury by inhibiting the $\mathrm{NF}-\kappa \mathrm{B}$ signaling pathway.
\end{abstract}

Correspondence to: Dr Haicheng Yuan, The Second Department of Neurology, Qingdao Central Hospital, 127 Siliu South Road, Qingdao, Shandong 266042, P.R. China

E-mail: h500592@163.com

Key words: microRNA-183, cerebral ischemia, microglia, nuclear factor- $\kappa \mathrm{B}$

\section{Introduction}

Ischemia stroke is the third leading cause of mortality and the major cause of disability worldwide $(1,2)$. The cause of ischemia stroke is the obstruction of cerebral blood flow with ischemia-reperfusion injury serving as its principal cause $(3,4)$. Following the onset of ischemia stroke, local brain tissue becomes necrotic and apoptotic, which leads to the corresponding neurological degeneration (5). Despite decades of research focusing on ischemia stroke, the therapeutic strategies for the treatment of this disease remain limited. Currently, the main treatment methods for ischemia stroke are thrombolytic therapy and management of symptoms (6). A previous study indicated that the immune response and inflammation serve important roles in brain tissue damage and the pathogenesis of ischemia stroke (7). Therefore, targeting ischemia stroke-induced neuroinflammation can be explored as a potential therapeutic option for this disease.

MicroRNAs (miRNAs) are small non-coding RNAs that are the key mediators for post-transcriptional gene silencing; which is achieved by binding to the 3'untranslated regions of their target mRNAs (8). A total of $30 \%$ of all mammalian protein-encoding genes are regulated by miRNAs $(9,10)$, and have been demonstrated to serve important functions in biological processes, including cell proliferation, differentiation, apoptosis, cell cycle and cellular stress response $(11,12)$. Accumulating evidence shows that miRNAs play critical roles in ischemia (13-15). The expression of various miRNAs has been found to be altered in the blood and brain of mammalian cells after the onset of stroke (16-19). MiRNA expression can be modulated by the application of external agents to alleviate stroke-induced loss of biological function $(16,20,21)$. In addition, prior mechanistic studies have suggested that miRNAs regulate the intracellular signaling of different inflammatory mediators, thus performing a neuroprotective role in ischemia stroke $(22,23)$. However, the underlying mechanisms of miRNA-mediated regulation of gene expression in relation to inflammation and the signaling pathways involved in this process remain poorly defined.

miR-183 was found to be expressed in the retina, and its expression decreases after maturation (24). In zebrafish, 
miR-183 regulates circadian rhythm by directly targeting E4 binding protein 4-6 and arylalkylamine $\mathrm{N}$-acetyltransferase 2 (25). Aberrant expression of miR-183 has been illustrated to contribute to the symptoms of neurodegenerative disorder in a mouse model (26). Indeed, downregulation of miR-183 has been reported in ectopic and eutopic tissues, and aberrant miR-183 expression may be associated with the development of endometriosis (27). Previously, miR-183 has been reported to be downregulated following stroke onset (28). In addition, depletion of miR-183 has been suggested to lead to microglia activation (29). However, the biological function and the mechanism of miR-183 expression associated with cerebral ischemia-reperfusion injury remains largely unknown. Therefore, the present study focuses on exploring the role and function of miR-183 in a rat model of cerebral ischemia-reperfusion injury by using miR-183 agomir, and investigating the underlying molecular mechanism.

\section{Materials and methods}

Experimental animals. A total of 36 specific pathogen-free (SPF) male Sprague-Dawley rats (8-10 weeks old; 250 $\pm 30 \mathrm{~g}$ ) were purchased from Jinan Peng Yue Experimental Animal Breeding Co., Ltd. [license number, SCXK (Lu) 2014-0007; Shandong, China]. The animals received food and water ad libitum, and were housed at a temperature of $23 \pm 2^{\circ} \mathrm{C}$, a humidity of $55 \pm 5 \%$ and a $12 / 12 \mathrm{~h}$ of light/dark cycle. Animal experiments were performed under NIH guidelines (No, 85-23; revised 1996) and approved by the Qingdao Central Hospital Animal Protection and Use Committee.

Establishment of cerebral ischemia-reperfusion model and animal groups. Rats were anesthetized by intraperitoneal injection of $3 \%$ sodium pentobarbital $(50 \mathrm{mg} / \mathrm{kg})$. Establishment of middle cerebral artery occlusion (MCAO) model was performed using the modified nylon suture method (1). Following $2 \mathrm{~h}$ of ischemia onset, the suture was gently pulled to the distal/proximal end of the external carotid artery before the rats were subsequently reperfused for $24 \mathrm{~h}$. The sham-operated group was destined for the separation and ligation of the blood vessels, and therefore the insertion of suture was not performed in this group. In accordance with the protocol described by Longa et al (30), the animals were scored $24 \mathrm{~h}$ after regaining consciousness and before the neurological deficits were recorded. The scores were specified as follows: 0 point (no symptoms of nerve damage were identified), 1 point (the contralateral forelimbs could not be fully extended when the tail of the animals was lifted), 2 points (turned to the temporal side when walking), 3 points (rats fell to the opposite side of the lesion when walking) and 4 points (rats could not walk spontaneously and lost their consciousness). An investigator who was blind to the experimental setup performed the analysis. A total of 36 rats were randomly divided into three separate groups: Sham operation (Control), MCAO and $\mathrm{MCAO}+$ miR-183 agomir groups. Intracerebroventrivular injection of miR-183 agomir (Guangzhou Ruibo Biotechnology Co., Ltd.) for $2 \mathrm{~h}$ after ischemia onset was performed in the $\mathrm{MCAO}+$ miR-183 agomir group, with the concentration at $20 \mu \mathrm{mol} / 1$ and total volume of $10 \mu \mathrm{l}$. The sham and MCAO groups were injected with corresponding amount of normal saline.

Analysis of miR-183 mRNA expression in brain tissue by reverse transcription (RT)-quantitative PCR. Following neurological function scoring, the rats were anesthetized by intraperitoneal injection of $3 \%$ sodium pentobarbital $(50 \mathrm{mg} / \mathrm{kg})$ and sacrificed by cervical dislocation. The cerebellum and lower brainstem were rapidly removed from the brain on ice, and the cerebral cortex of the ischemic tissues of 6 rats in each group were placed in liquid nitrogen for subsequent experiments. Tissues $(50 \mathrm{mg})$ were ground into powder with liquid nitrogen, in a mortor, and centrifuged at 1,204 x g for $15 \mathrm{~min}$ at $4^{\circ} \mathrm{C}$. According to the manufacturer's protocol, total RNA was extracted using TRIzol (Thermo Fisher Scientific, Inc.). Absorption ratios at $260 / 280 \mathrm{~nm}$ of 1.8 and 2.0 were used to evaluate RNA purity. SuperScript ${ }^{\mathrm{TM}}$ IV Reverse Transcriptase (cat. no. 18090010; Thermo Fisher Scientific, Inc.) was used for the reverse transcription of RNA into cDNA at $65^{\circ} \mathrm{C}$, according to the manufacture's instruction. RT-qPCR was performed using SYBR Green I (Thermo Fisher Scientific, Inc.) and a Mastercycler ${ }^{\circledR}$ nexus X2 (Eppendorf). The thermocycling conditions were as follows: $95^{\circ} \mathrm{C}$ for $10 \mathrm{~min}, 95^{\circ} \mathrm{C}$ for $10 \mathrm{sec}$ ( 40 cycles), $60^{\circ} \mathrm{C}$ for $1 \mathrm{~min}$. Results were quantified using the $2^{-\triangle \Delta \mathrm{Cq}}$ method (31). The relative expression levels of miR-183 were calculated using let-7a as internal control. The following primers were used for RT-qPCR: miR-183, forward, 5'-AGGAGCAGAGGAGGTCTTT-3' and reverse, 5'-TATGGC ACTGGTAGAATTCACT-3'.

Tetrazolium chloride (TTC) staining. The rats were anesthetized by intraperitoneal injection of $3 \%$ sodium pentobarbital $(50 \mathrm{mg} / \mathrm{kg}$ ) and subsequently sacrificed by cervical dislocation after neurological functions were scored. Brain tissue was placed on ice, before the brain tissues of 6 rats in each group were frozen at $-20^{\circ} \mathrm{C}$ for $30 \mathrm{~min}$. The brain tissue was then cut into coronal sections, which were quickly placed in $2 \%$ TTC (cat. no. G3005; Beijing Solarbio Science \& Technology Co., Ltd.) solution for incubation at $37^{\circ} \mathrm{C}$ for $15 \mathrm{~min}$, before placement in $4 \%$ paraformaldehyde for fixation for $24 \mathrm{~h}$, at $25^{\circ} \mathrm{C}$. The tissue was washed with PBS for 3-5 min, and images were captured using a x100 optical microscope (Olympus Corporation). The image analysis software Image $\mathrm{J}$ 1.43 (National Institutes of Health) was applied to measure the volume of cerebral infarction.

Analysis of ionized calcium-binding adaptor molecule 1 (IBA-1)-positive cells by immunohistochemistry. Following TTC staining, the brain tissues were dehydrated and embedded in paraffin, before being serially sliced to a thickness of $5 \mu \mathrm{m}$. After dewaxed in xylene, the brain tissue sections were rehydrated in a descending ethanol gradient, at room temperature. At room temperature, the sections were subsequently inactivated with a $3 \% \mathrm{H}_{2} \mathrm{O}_{2}$ solution in methanol for $20 \mathrm{~min}$, citrate buffer ( $\mathrm{pH} \mathrm{6.0)}$ at $65^{\circ} \mathrm{C}$ for $10 \mathrm{~min}$, before incubation with $5 \%$ bovine serum albumin (cat.no. A8020; Beijing Solarbio Science $\&$ Technology Co., Ltd.) at $25^{\circ} \mathrm{C}$ for $20 \mathrm{~min}$. The sections were then incubated using primary rabbit anti-rat IBA-1 polyclonal antibody (1:200; orb336635; Biorbyt Ltd.) at $4^{\circ} \mathrm{C}$ overnight. After PBS washing, the sections were incubated 
with horseradish peroxidase-conjugated goat anti-rabbit IgG secondary antibody (1:1,000; ABIN101988; antibodies-online $\mathrm{GmbH}$, Aachen) at $37^{\circ} \mathrm{C}$ for $30 \mathrm{~min}$. Following secondary antibody incubation, 3,3'-diaminobenzidine (DAB) staining was developed on the sections for 5-10 $\mathrm{min}$ at room temperature, before counterstaining with hematoxylin for $10 \mathrm{~min}$ at $37^{\circ} \mathrm{C}$. Finally, the sections were dehydrated by gradient alcohol for $5 \mathrm{~min}$, treated twice with xylene for $10 \mathrm{~min}$, before being sealed with mounting medium.

The results were observed under a 4400 optical microscope (Olympus Corporation) and counted using Aperio Imagescope 11.1 software (Leica Microsystems Inc.). The percentage (\%) of positive cells was evaluated.

Evaluation of $I L-1 \beta, I L-6$ and TNF- $\alpha$ expression in brain tissue by ELISA. Brain tissue was thoroughly homogenized in ice cold PBS, before centrifugation at $500 \mathrm{x} \mathrm{g}$ at $4^{\circ} \mathrm{C}$ for $15 \mathrm{~min}$. The supernatant was then assayed for IL-1 $\beta$ (orb79117; Biorbyt Ltd.), IL-6 (orb79123; Biorbyt Ltd.) and TNF- $\alpha$ (orb79138-480; Biorbyt Ltd.) according to manufacturer's protocols. The results were obtained by measuring absorption at $450 \mathrm{~nm}$ on a microplate reader (Model 680, Bio-Rad Laboratories, Inc.).

Western blot analysis of $N F-\kappa B$-associated protein expression in brain tissue. The cerebral cortex of the ischemic area was grounded and homogenized before centrifugation $(800 \mathrm{x} \mathrm{g}$; $10 \mathrm{~min} ; 4^{\circ} \mathrm{C}$ ), and the supernatant was extracted. The bicinchoninic assay (BCA) kit (Beijing Solarbio Science \& Technology Co., Ltd.) was used to quantify protein concentration. A total of $40 \mu \mathrm{g}$ of each protein sample was mixed with $10 \%$ of the SDS gel buffer at a 1:1 ratio. This mixture was then boiled at $95^{\circ} \mathrm{C}$ for $5 \mathrm{~min}$. Following denaturation, the protein samples were separated by SDS-PAGE (10\% gels) and transferred onto a polyvinylidene membrane (Merck KGaA, Darmstadt) at $80 \mathrm{~V}$ for $30 \mathrm{~min}$. The membranes were subsequently blocked with 5\% skim milk powder dissolved in TBS with Tween-20 (TBS-T) for $1 \mathrm{~h}$ at $4^{\circ} \mathrm{C}$, before incubation with their respective rabbit anti-rat polyclonal antibodies dissolved in TBS-T solution containing $3 \%$ bovine serum albumin at $4{ }^{\circ} \mathrm{C}$ overnight. The antibodies used in this study were as follows: NF- $\kappa \mathrm{B}$ p65 (1:500; orb11118; Biorbyt Ltd.), IкB $\alpha$ (1:500; orb223182; Biorbyt Ltd.) and $\beta$-actin (1:2,000; orb178392; Biorbyt Ltd.). The membranes were then washed with TBS-T four times prior to incubation with horseradish peroxidase-conjugated goat anti-rabbit IgG (1:1,000; ABIN101988; antibodies-online $\mathrm{GmbH}$ ) at room temperature for $1 \mathrm{~h}$. The membranes were subsequently washed with TBS-T four times and incubated with ECL luminescent substrate (Thermo Fisher Scientific, Inc.) for 3-5 min for visualization. Levels of protein expression were normalized to $\beta$-actin, and quantification was performed using Image J software (v 1.51; National Institutes of Health).

Statistical analysis. All data was processed using the SPSS 19.0 statistical analysis software (IBM Corp.). Data analysis was expressed as mean \pm standard deviation (mean $\pm \mathrm{SD}$ ), and comparison between groups was performed using one-way analysis of variance (ANOVA) followed by least significance difference (LSD) test. $\mathrm{P}<0.05$ was considered to indicate a statistically significant difference.

\section{Results}

miR-183 agomir treatment improves neurological function in rat brain tissue after ischemia-reperfusion injury. Neurological function was scored in rats following cerebral ischemia onset. The control rats exhibited a neurological score of zero when comparing with the MCAO group, which was used as a positive control. The neurological scores of the MCAO + miR-183 agomir group were significantly reduced compared with those in the MCAO group (Fig. 1A). The effect of miR-183 agomir on miR-183 mRNA levels of brain tissue was measured using RT-qPCR. Compared with the control group, the expression of miR-183 mRNA in the brain tissue of rats in MCAO and MCAO + miR-183 agomir groups was significantly reduced (Fig. 1B). Following miR-183 agomir treatment, the expression of miR-183 mRNA was significantly increased in the brain tissue of rats compared with those in the MCAO group (Fig. 1B).

The effect of miR-183 on brain tissue damage was determined by TTC staining following cerebral ischemia induction in rats, with the non-infarcted areas staining red. The percentage of cerebral infarction volume in the MCAO + miR-183 agomir group appeared to be significantly lower compared with that in the MCAO group (Fig. 2). This observation suggests that miR-183 agomir treatment reduced brain tissue damage in rats with cerebral ischemia reperfusion. These results collectively indicate that cerebral miR-183 expression is reduced by ischemia onset, an effect that can be partially rescued by miR-183 agomir treatment. Subsequent miR-183 agomir treatment increased miR-183 expression and improved neurological function, suggesting that miR-183 serves a neuroprotective role in rats with cerebral ischemia reperfusion.

miR-183 agomir treatment decreases the expression of IBA-1 in rats with cerebral ischemia-reperfusion injury. It has been reported that IBA-1 expression may be increased in rat brain following the onset of cerebral ischemia (32). As a result, IBA-1 expression levels and the distribution of IBA-1-positive cells in the three treatment groups were assessed using immunohistochemistry. Whilst only a small number of IBA-1-positive cells (arrows) were found in the hippocampal CA1 of the control group (Fig. 3A and D), a large number of IBA-1-positive cells appeared in the CA1 region in the MCAO group (Fig. 3B and D). The number of IBA-1-positive cells observed in the hippocampal CA1 area was significantly reduced following miR-183 agomir treatment (Fig. 3C and D), when compared with the MCAO group. This finding suggests that miR-183 agomir treatment decreases the expression of IBA-1 in rats following cerebral ischemia-reperfusion injury.

miR-183 agomir treatment decreases the expression of inflammatory cytokines $I L-1 \beta, I L-6$ and TNF- $\alpha$ in brain tissue. Results from the ELISA assay demonstrated that the expression of inflammatory factors IL- $1 \beta$, IL- 6 and TNF- $\alpha$ in the brain tissues of MCAO and MCAO + miR-183 agomir groups were significantly increased following the cerebral ischemia onset compared with the control group (Fig. 4A-C). After treatment with miR-183 agomir, the expressions of IL-1 $\beta$, IL- 6 and TNF- $\alpha$ were significantly decreased in the brain tissue of rats when compared with MCAO group (Fig. 4A-C). These results implicate miR-183 to be a suppressor of inflammation during cerebral ischemia. 
A

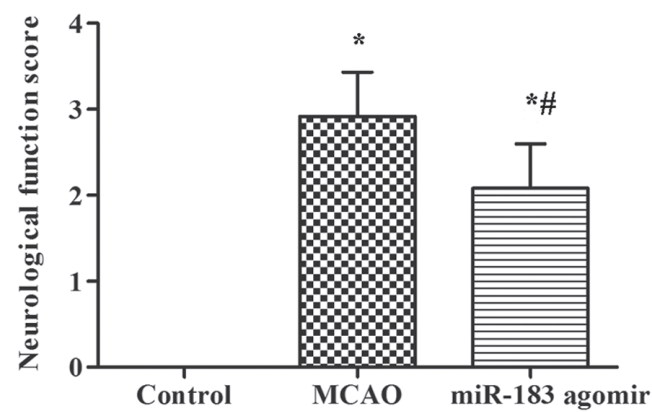

B

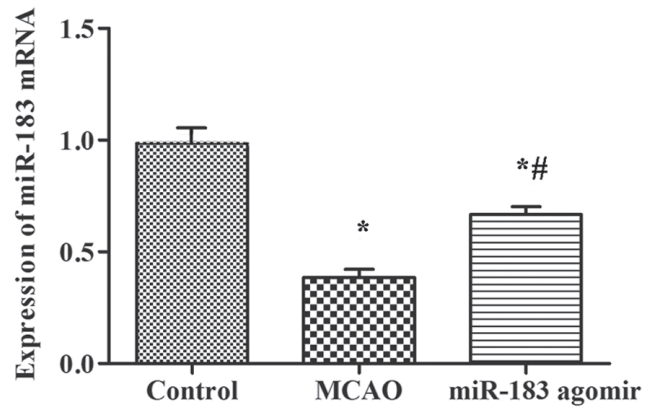

Figure 1. Neurological function scores in rats from control, MCAO and $\mathrm{MCAO}+$ miR-183 agomir groups and expression levels of miR-183 mRNA in brain tissue. (A) Neurological function score was measured in control, MCAO and MCAO + miR-183 agomir groups. (B) Reverse transcription-qPCR was used to detect the expression of miR-183 mRNA in brain tissue in control, $\mathrm{MCAO}$ and $\mathrm{MCAO}+$ miR-183 agomir groups. ${ }^{*} \mathrm{P}<0.05$ vs. control group; ${ }^{\text {"}} \mathrm{P}<0.05$ vs. the MCAO group. miR-183, microRNA-183; MCAO, middle cerebral artery occlusion.

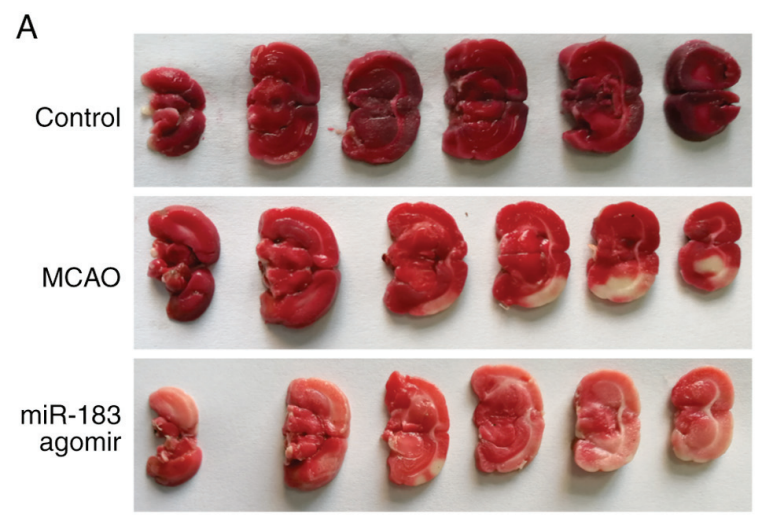

B

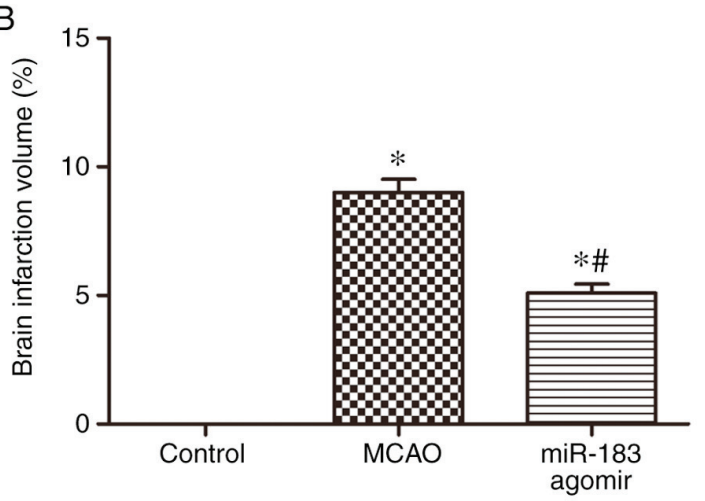

Figure 2. Tetrazolium Chloride (TTC) staining in rat brain tissue. (A) The infarcted area of the brain tissue was stained following the induction of cerebral ischemia in rats using TTC staining. (B) The percentage of cerebral infarction volume was quantified and analyzed. ${ }^{*} \mathrm{P}<0.05$ vs. control group; ${ }^{\#} \mathrm{P}<0.05$ vs. the MCAO group. miR-183, microRNA-183; MCAO, middle cerebral artery occlusion.
miR-183 agomir treatment inhibits $N F-\kappa B$ signaling in brain tissue. Lastly, the effect of miR-183 on the regulation of $\mathrm{NF}-\kappa \mathrm{B}$ signaling was investigated by measuring the protein levels of the NF- $\kappa \mathrm{B}$ subunit $\mathrm{p} 65$ and $\mathrm{I} \kappa \mathrm{B} \alpha$, a negative regulator of $\mathrm{NF}-\kappa \mathrm{B}$ (33), in the brain tissues of the three treatment groups. The expression of p65 in the brain tissue of MCAO and $\mathrm{MCAO}+$ miR-183 agomir groups was significantly increased compared with the control group, while the expression of $\mathrm{I} \kappa \mathrm{B} \alpha$ was significantly decreased (Fig. 5). In contrast, the expression of p65 in brain tissue of MCAO + miR-183 agomir group was significantly decreased, and the expression of $\mathrm{I} \kappa \mathrm{B} \alpha$ was significantly increased when compared with MCAO group (Fig. 5). This finding suggests that miR-183 agomir treatment inhibits $\mathrm{NF}-\kappa \mathrm{B}$ signaling activation.

\section{Discussion}

miRNAs serve important roles in a number of pathophysiological conditions by the virtue of binding to and regulating target gene expression. Previously, a number of studies reported that miRNAs can mediate ischemia injury, the suppression of which by treatment with their respective mimic significantly alleviated brain tissue damage in ischemia stroke models $(34,35)$. Indeed, Wang et al (35) showed that miR-3473b expression was significantly increased, and treatment with miR-3473b antagomir significantly reduced infarction after MCAO in mice. In another study, Xiang et al (29) found that following ischemic preconditioning for $15 \mathrm{~min}$, the expression of $\mathrm{miR}-183$ was demonstrated to be upregulated after 3 and $24 \mathrm{~h}$ reperfusion. However, the role and biological function of miR-183 remains unknown. In the present study, the role of miR-183 in ischemia reperfusion-induced brain damage was investigated by employing a model of cerebral ischemia-reperfusion injury. Upon treatment with miR-183 agomir, the neurological score was markedly decreased in rats with cerebral ischemia-reperfusion injury. On the molecular level, results from the present study indicated that miR-183 expression was significantly decreased in rats with cerebral ischemia-reperfusion injury, an effect that was partially rescued by miR-183 agomir treatment. Therefore, these findings suggest a neuroprotective role of miR-183 agomir in the cerebral ischemia model, which correlate well with previous studies (29).

Cerebral infarction volume is a long established measure of outcome in clinical trials of acute ischemia stroke therapies $(36,37)$. Following miR-183 agomir treatment, the cerebral infarction volume profoundly decreased compared with the MCAO group, which indicated that tissue damage was alleviated by miR-183 agomir treatment. IBA-1, a calcium binding protein, which is specifically expressed in microglia, has been reported to be a critical factor in microglia membrane ruffling, as well as being involved in the regulation of Rho-GTPase, Rac and calcium signaling pathways, and is required for cell mobility and phagocytosis $(38,39)$. In addition, high levels of IBA-1 expression in activated microglia suggest that IBA may be a potential marker for microglia activation (40). Thus, it serves as one of the most useful proteins for distinguishing microglia from other types of brain tissues in studies of cerebral ischemia $(38,41)$. In 

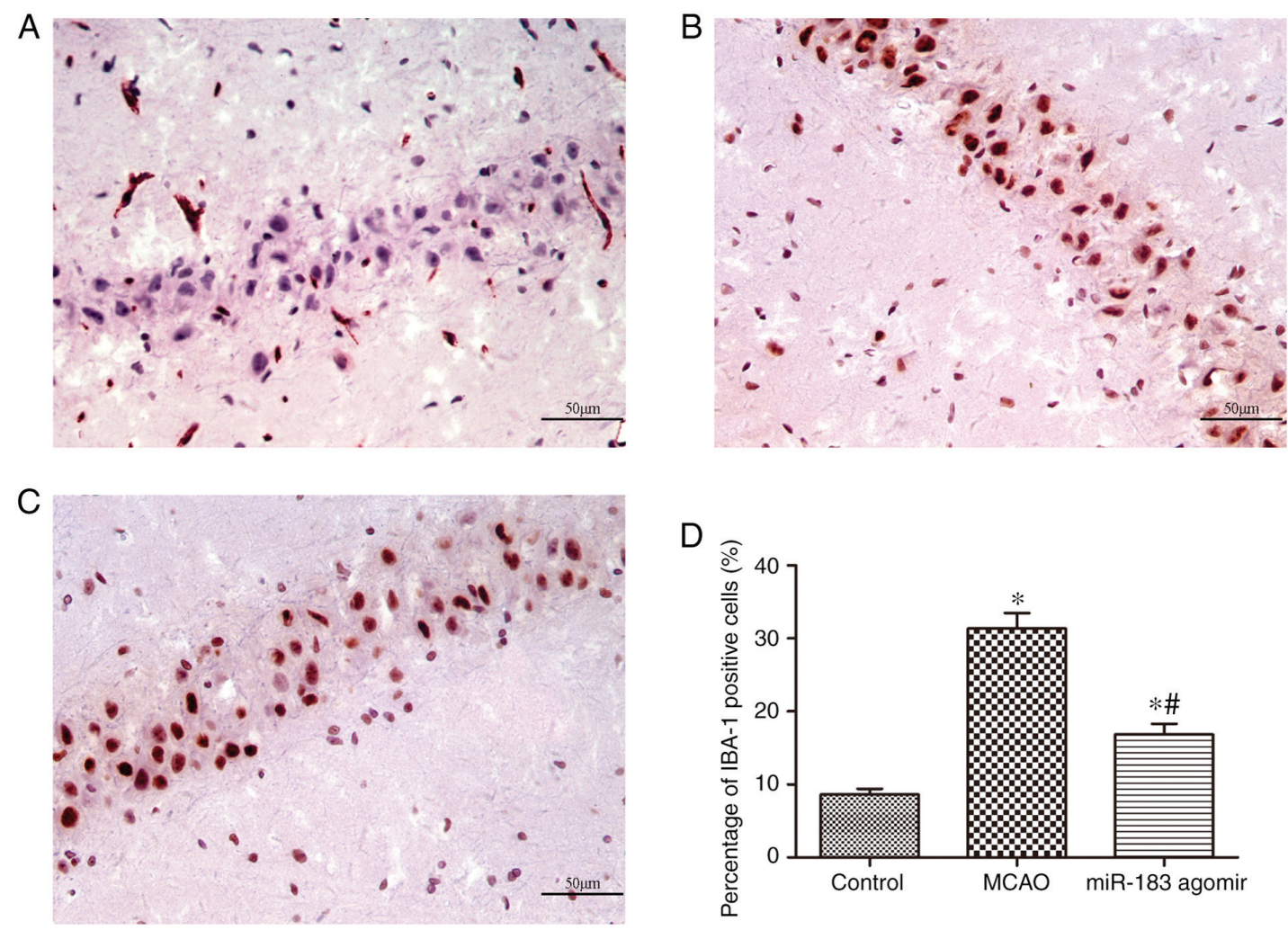

Figure 3. Evaluation of IBA-1-positive cells by immunohistochemistry. Immunohistochemistry was performed to stain and visualize IBA-1-positive cells in the hippocampal CA1 region of rats in (A) control, (B) MCAO and (C) MCAO + miR-183 agomir groups. (D) The number of IBA-1-positive cells in the three treatment groups was quantified and analyzed. " $\mathrm{P}<0.05$ vs. control group; ${ }^{*} \mathrm{P}<0.05$ vs. MCAO group. IBA-1, ionized calcium-binding adapter molecule 1 ; miR-183, microRNA-183; MCAO, middle cerebral artery occlusion.
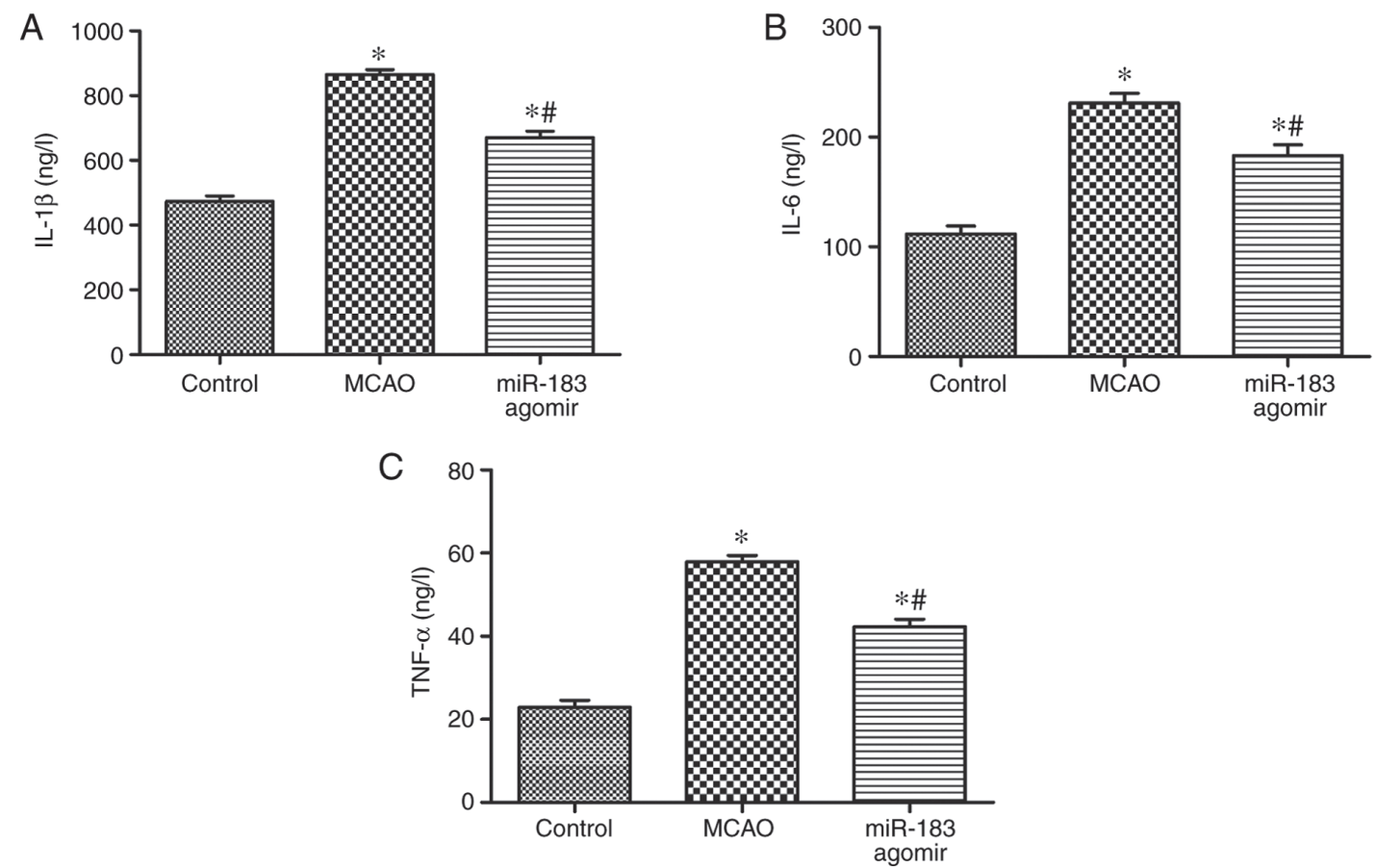

Figure 4. Expression of inflammatory factors IL-1 $\beta$, IL- 6 and TNF- $\alpha$ in brain tissues. The protein levels of (A) IL-1 $\beta$, (B) IL-6 and (C) TNF- $\alpha$ in brain tissue in each group was determined using ELISA. "P<0.05 vs. control group; ${ }^{\#} \mathrm{P}<0.05$ vs. MCAO group. IL-1 $\beta$, interleukin- $1 \beta$; IL-6, interleukin-6; TNF- $\alpha$, tumor necrosis factor- $\alpha$; miR-183, microRNA-183; MCAO, middle cerebral artery occlusion.

this study, immunohistochemistry staining results showed that the number of IBA-1-positive cells was significantly decreased in rats treated with miR-183 agomir after cerebral ischemia-reperfusion injury. 


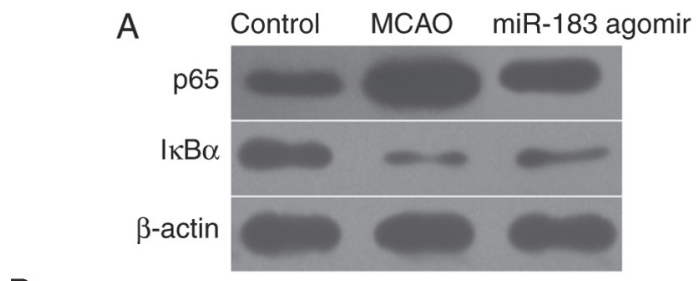

B

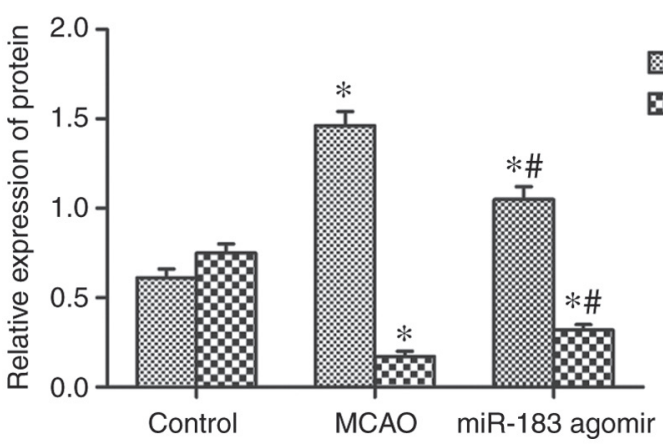

Figure 5. Western blot analysis of NF- $\mathrm{kB}$-associated protein expression in rat brain tissue. (A) Protein expression levels of $\mathrm{p} 65$ and $\mathrm{I \kappa B} \alpha$ in the brain tissue from each group were determined by western blot analysis. $\beta$-actin expression level was used as reference. (B) The expression of p65 and IкB $\alpha$ was quantified and analyzed. ${ }^{*} \mathrm{P}<0.05$ vs. control group; ${ }^{~} \mathrm{P}<0.05$ vs. $\mathrm{MCAO}$ group. NF- $\kappa B$, Nuclear Factor- $\kappa \mathrm{B}$; I $\kappa \mathrm{B} \alpha$, nuclear factor of $\kappa$ light polypeptide gene enhancer in $\mathrm{B}$-cells inhibitor, alpha; $\mathrm{MCAO}$, middle cerebral artery occlusion.

Next, the mechanism involved in the neuroprotection of miR-183 agomir in cerebral ischemia-reperfusion rat models was investigated. Inflammation and immune response serve a pivotal role in stroke induced by tissue damage and repair (42). Numerous studies have reported that the expression level of miRNAs was altered following ischemia stroke onset $(13,43,44)$. Around 50 miRNAs have been found to be upregulated in the rat model of ischemic stroke, a list which includes but not limited to miR-17, miR-134 and miR-206 (16-20). On the other hand, several miRNAs including miR-25, miR-138 and miR-361 were found to be downregulated in a rat model of ischemia (43). These miRNAs regulate the intracellular pathways of a variety of inflammatory mediators (43). In the present study, the effect of miR-183 agomir on the expression of IL-6, IL- $1 \beta$ and TNF- $\alpha$, three prominent pro-inflammatory cytokines (35), was assessed. ELISA results reported that the expression of all three were increased following the induction of cerebral ischemia-reperfusion injury, an observation that was partially reversed by miR-183 agomir treatment. Thus, these results suggest that miR-183 agomir-mediated enhancement of neurological function and reduction of tissue damage is at least in part due to the downregulation of pro-inflammatory cytokine expression.

The toll-like receptor 4 (TLR4)/NF-кB signaling pathway was reported to be activated in cerebral ischemia-reperfusion injury in rats (33). In this aforementioned study, the expression of TLR4 and NF- $\mathrm{kB}$ was found to be increased along with the activation of corresponding signaling pathway following cerebral ischemia-reperfusion injury. Data from the present study supports these results, the expression of p65 and IкB $\alpha$ in rats with cerebral ischemia-reperfusion was evaluated. Furthermore, miR-183 agomir treatment partially reversed this effect. Altogether, this study revealed that miR-183 regulates the activation of microglia in cerebral ischemia-reperfusion injury in rats by inhibiting NF- $\kappa B$ signaling pathway. Therefore, the present study uncovers a role for miR-183 in the development of cerebral ischemia reperfusion-injury in rats, along with further mechanistic insights. This information may be useful for further clinical studies and treatment strategies of patients with ischemia stroke. Thus, miR-183 agomir can be explored as a potential therapeutic reagent for treatment of ischemia stroke in the future clinically. A potential limitation of this study is that only one concentration of miR-183 agomir was tested. In future investigations, different concentrations will be tested in vitro and in vivo to improve our understanding of miR-183 as a therapeutic target.

The results of the current study indicated that miR-183 agomir treatment significantly reduced neurological function scores, and serves a neuroprotection role by reducing the percentage of cerebral infarction volume, and decreasing the IBA-1-positive cells in the CA1 area of the hippocampus in response to rat cerebral ischemia-reperfusion injury. Furthermore, miR-183 agomir treatment decreased the expression of pro-inflammatory proteins and regulated the activation of microglia in cerebral ischemia-reperfusion injury in rats by inhibiting NF- $\mathrm{kB}$ signaling pathway. Therefore, these results uncover the role of miR-183 in cerebral ischemia-reperfusion. miR-183 may be used as a therapeutic target for the treatment of cerebral ischemia-reperfusion.

\section{Acknowledgements}

Not applicable.

\section{Funding}

The present study was supported by National Major Special Projects for the Major New Drug Creation (grant no. 2012ZX09103-101-015).

\section{Availability of data and materials}

All data generated or analyzed during this study are included in this published article.

\section{Authors' contributions}

BX, PZ and HY conceived and designed the study. LF, XW, and YS performed the experiments. BX wrote the paper. PZ reviewed and edited the manuscript. All authors read and approved the manuscript.

\section{Ethics approval and consent to participate}

Animal experiments were performed under NIH guidelines (publication no. 85-123; revised 1996) and approved by the Qingdao Central Hospital Animal Protection and Use Committee.

\section{Patient consent for publication}

Not applicable. 


\section{Competing interest}

The authors declare that they have no competing interests.

\section{References}

1. Mendis S, Davis S and Norrving B: Organizational update: The world health organization global status report on noncommunicable diseases 2014; One more landmark step in the combat against stroke and vascular disease. Stroke 46 : e121-122, 2015

2. Zhang R, Zhang Z and Chopp M: Function of neural stem cells in ischemic brain repair processes. J Cereb Blood Flow Metab 36 2034-2043, 2016.

3. Schaller B and Graf R: Cerebral ischemia and reperfusion: The pathophysiologic concept as a basis for clinical therapy. J Cereb Blood Flow Metab 24: 351-371, 2004.

4. Baird AE, Donnan GA, Austin MC, Fitt GJ, Davis SM and McKay WJ: Reperfusion after thrombolytic therapy in ischemic stroke measured by single-photon emission computed-tomography. Stroke 25: 79-85, 1994.

5. Lee JM, Grabb MC, Zipfel GJ and Choi DW: Brain tissue responses to ischemia. J Clin Invest 106: 723-731, 2000.

6. Blakeley JO and Llinas RH: Thrombolytic therapy for acute ischemic stroke. J Neurol Sci 261: 55-62, 2007.

7. Khoshnam SE, Winlow W, Farzaneh M, Farbood Y and Moghaddam HF: Pathogenic mechanisms following ischemic stroke. Neurol Sci 38: 1167-1186, 2017.

8. Zhang CX: MicroRNomics: A newly emerging approach for disease biology. Physiol Genomics 33: 139-147, 2008.

9. Friedman RC, Farh KK, Burge CB and Bartel DP: Most mammalian mRNAs are conserved targets of microRNAs. Genome Res 19: 92-105, 2009

10. Griffiths-Jones S, Saini HK, van Dongen S and Enright AJ: miRBase: Tools for microRNA genomics. Nucleic Acids Res 36 (Database Issue): D154-D158, 2008.

11. Kloosterman WP and Plasterk RH: The diverse functions of MicroRNAs in animal development and disease. Dev Cell 11: 441-450, 2006

12. Felekkis K, Touvana E, Stefanou $\mathrm{CH}$ and Deltas C: microRNAs: A newly described class of encoded molecules that play a role in health and disease. Hippokratia 14: 236-240, 2010.

13. Ouyang YB, Stary CM, Yang GY and Giffard R: microRNAs: Innovative targets for cerebral ischemia and stroke. Curr Drug Targets 14: 90-101, 2013.

14. Saugstad JA: MicroRNAs as effectors of brain function with roles in ischemia and injury, neuroprotection, and neurodegeneration. J Cereb Blood Flow Metab 30: 1564-1576, 2010.

15. Li GW, Morris-Blanco KC, Lopez MS, Yang T, Zhao H, Vemuganti R and Luo Y: Impact of microRNAs on ischemic stroke: From pre- to post-disease. Prog Neurobiol 163-164: 59-78, 2018.

16. Jeyaseelan K, Lim KY and Armugam A: MicroRNA expression in the blood and brain of rats subjected to transient focal ischemia by middle cerebral artery occlusion. Stroke 39: 959-966, 2008.

17. Dharap A, Bowen K, Place R, Li LC and Vemuganti R: Transient focal ischemia induces extensive temporal changes in rat cerebral MicroRNAome. J Cereb Blood Flow Metab 29: 675-687, 2009.

18. Sørensen SS, Nygaard AB, Nielsen MY, Jensen K and Christensen T: miRNA expression profiles in cerebrospinal fluid and blood of patients with acute ischemic stroke. Transl Stroke Res 5: 711-718, 2014.

19. Li SH, Su SY and Liu JL: Differential regulation of microRNAs in patients with ischemic stroke. Curr Neurovasc Res 12: 214-221, 2015.

20. Saugstad JA: Non-coding RNAs in stroke and neuroprotection. Front Neurol 6: 50, 2015.

21. Liu da Z, Jickling GC, Ander BP, Hull H, Zhan X, Cox C, Shroff N, Dykstra-Aiello C, Stamova B and Sharp FR: Elevating microRNA-122 in blood improves outcomes after temporary middle cerebral artery occlusion in rats. J Cereb Blood Flow Metab 36: 1374-1383, 2016.

22. Khoshnam SE, Winlow W and Farzaneh M: The Interplay of MicroRNAs in the inflammatory mechanisms following ischemic stroke. J Neuropathol Exp Neurol 76: $548-561,2017$
23. Liu P, Zhao H, Wang R, Wang P, Tao Z, Gao L, Yan F, Liu X, Yu S, Ji X and Luo Y: MicroRNA-424 protects against focal cerebral ischemia and reperfusion injury in mice by suppressing oxidative stress. Stroke 46: 513-519, 2015.

24. Dambal S, Shah M, Mihelich B and Nonn L: The microRNA-183 cluster: The family that plays together stays together. Nucleic Acids Res 43: 7173-7188, 2015.

25. Wienholds E, Kloosterman WP, Miska E, Alvarez-Saavedra E, Berezikov E, de Bruijn E, Horvitz HR, Kauppinen S and Plasterk RH: MicroRNA expression in zebrafish embryonic development. Mechanisms Dev 122 (Suppl): S149-S150, 2005.

26. Kye MJ, Niederst ED, Wertz MH, Gonçalves Ido C, Akten B, Dover KZ, Peters M, Riessland M, Neveu P, Wirth B, et al: SMN regulates axonal local translation via miR-183/mTOR pathway. Hum Mol Genet 23: 6318-6331, 2014.

27. Shi XY, Gu L, Chen J, Guo XR and Shi YL: Downregulation of miR-183 inhibits apoptosis and enhances the invasive potential of endometrial stromal cells in endometriosis. Int J Mol Med 33: 59-67, 2014.

28. Sepramaniam S, Jun-Rong T, Kay-Sin T, Deidre Ann De S, Subramaniam T, Fung-Peng W, Chee-Woon W, Fung-Lin Y, Dwi-Setyowati K, Prameet K, et al: Circulating MicroRNAs as Biomarkers of Acute Stroke. Int J Mol Sci 15: 1418-1432, 2014.

29. Xiang L, Chen XJ, Wu KC, Zhang CJ, Zhou GH, Lv JN, Sun LF, Cheng FF, Cai XB and Jin ZB: miR-183/96 plays a pivotal regulatory role in mouse photoreceptor maturation and maintenance. Proc Natl Acad Sci USA 114: 6376-6381.

30. Longa EZ, Weinstein PR, Carlson S and Cummins R: Reversible middle cerebral artery occlusion without craniectomy in rats. Stroke 20: 84-91, 1989.

31. Livak KJ and Schmittgen TD: Analysis of relative gene expression data using real-time quantitative PCR and the 2(-Delta Delta C(T)) method. Methods 25: 402-408, 2001.

32. Ito D, Tanaka K, Suzuki S, Dembo T and Fukuuchi Y: Enhanced expression of Iba1, ionized calcium-binding adapter molecule 1 , after transient focal cerebral ischemia in rat brain. Stroke 32: 1208-1215, 2001.

33. Chen J, Yang C, Xu X, Yang Y and Xu B: The effect of focal cerebral ischemia-reperfusion injury on TLR4 and NF- $\kappa B$ signaling pathway. Exp Ther Med 15: 897-903, 2018.

34. Ni J, Wang X, Chen S, Liu H, Wang Y, Xu X, Cheng J, Jia J and Zhen X: MicroRNA let-7c-5p protects against cerebral ischemia injury via mechanisms involving the inhibition of microglia activation. Brain Behav Immun 49: 75-85, 2015.

35. Wang X, Chen S, Ni J, Cheng J, Jia J and Zhen X: miRNA-3473b contributes to neuroinflammation following cerebral ischemia. Cell Death Dis 9: 11, 2018.

36. van der Worp HB, Claus SP, Bär PR, Ramos LM, Algra A van Gijn J and Kappelle LJ: Reproducibility of measurements of cerebral infarct volume on CT scans. Stroke 32: 424-430, 2001.

37. Han M, Choi JW, Rim NJ, Kim SY, Suh HI, Lee KS, Hong JM and Lee JS: Cerebral infarct volume measurements to improve patient selection for endovascular treatment. Medicine (Baltimore) 95: e4702, 2016.

38. Imai Y, Ibata I, Ito D, Ohsawa $\mathrm{K}$ and Kohsaka S: A novel gene iba1 in the major histocompatibility complex class III region encoding an $\mathrm{EF}$ hand protein expressed in a monocytic lineage. Biochem Biophys Res Commun 224: 855-862, 1996.

39. Ohsawa K, Imai $Y$, Kanazawa $H$, Sasaki $Y$ and Kohsaka S: Involvement of Iba1 in membrane ruffling and phagocytosis of macrophages/microglia. J Cell Sci 113: 3073-3084, 2000.

40. Ito D, Imai Y, Ohsawa K, Nakajima K, Fukuuchi Y and Kohsaka S: Microglia-specific localisation of a novel calcium binding protein, Iba1. Brain Res Mol Brain Res 57: 1-9, 1998.

41. Ito D, Tanaka K, Suzuki S, Dembo T and Fukuuchi Y: Enhanced expression of Iba1, ionized calcium-binding adapter molecule 1, after transient focal cerebral ischemia in rat brain. Stroke 32: 1208-1215, 2001

42. Ma Y, Wang J, Wang Y and Yang GY: The biphasic function of microglia in ischemic stroke. Prog Neurobiol 157: 247-272, 2017.

43. Rink C and Khanna S: MicroRNA in ischemic stroke etiology and pathology. Physiol Genomics 43: 521-528, 2011.

44. Hamzei Taj S, Kho W, Riou A, Wiedermann D and Hoehn M: MiRNA-124 induces neuroprotection and functional improvement after focal cerebral ischemia. Biomaterials 91: 151-165, 2016.

This work is licensed under a Creative Commons Attribution-NonCommercial-NoDerivatives 4.0 International (CC BY-NC-ND 4.0) License. 